\title{
External Cephalic Version: A Single Centre Experience
}

\author{
Kassam Mahomed1,2,3, Poornima Amaranarayana1, Ibinabo Ibiebele ${ }^{3}$ \\ ${ }^{1}$ Department of Obstetrics and Gynaecology, Ipswich Hospital, Ipswich, Australia \\ ${ }^{2}$ University of Queensland, Brisbane, Australia \\ ${ }^{3}$ Mater Research Institute, South Brisbane, Australia \\ Email: kassam mahomed@health.qld.gov.au
}

Received 26 February 2014; revised 22 March 2014; accepted 29 March 2014

Copyright (C) 2014 by authors and Scientific Research Publishing Inc.

This work is licensed under the Creative Commons Attribution International License (CC BY). http://creativecommons.org/licenses/by/4.0/

(c) (i) Open Access

\begin{abstract}
Incidence of caesarean section for breech presentation has increased markedly. External cephalic version (ECV) is effective in reducing non cephalic presentation at births and caesarean section (CS) for breech presentation. Success rates are good but there is increasing need for induction of labour (IOL) as well as for intrapartum CS for reasons that are not clear. The aim of this study was to report the experience with ECV at a single centre where ECVs were performed by or under the guidance of one senior clinician. This was a 7-year retrospective audit of $147 \mathrm{ECVs}$ at a single centre in Queensland. ECV was successful in 53.7\%, 34\% in nulliparous and 69\% in multiparous women. Of the few variables explored, nulliparity was the only variable that was associated with poor success. Among those with successful ECV, 32 (40.5\%) had IOL. Intrapartum CS rate in women who had had a successful ECV was $16.5 \%$ compared to $11 \%$ for the hospital. There was no difference in early neonatal outcome between the groups. We have confirmed a success rate that is comparable with many other studies. Mothers with successful ECV do have a higher IOL and a higher CS rate especially in women whose labour is induced. We suggest a need to look at some novel methods to increase uptake further and to improve success rates in nulliparous women.
\end{abstract}

\section{Keywords}

ECV, Breech, Labour, Caesarean, Predictors

\section{Introduction}

Approximately 3\% - 4\% of pregnant women will present with a fetus in breech position at term [1]. Incidence of caesarean section (CS) for breech presentation has increased markedly in most well resourced countries since the publication of the term breech trial [2] [3]. It has clearly been shown [4] that external cephalic version (ECV) 
is effective in reducing non cephalic presentation at births as well as reducing the need for CS for breech presentation. Current guidelines [5]-[7] therefore recommend that ECV should be offered to all women with breech presentation at term. Success rates of ECV vary between $40 \%-60 \%$ [5] with it being more successful if multiparous, breech is not low in the pelvis, uterus not tense, easily palpable fetal head, and maternal weight less than $65 \mathrm{~kg}$ [8]. It is also likely that adequate amounts of amniotic fluid, non-frank breech, non-anterior placenta and anterior fetal back also improve success rate [9] [10].

The aim of this retrospective audit was to report the experience with ECV at a single centre where ECVs were performed by or under the guidance of one clinician with much experience with ECVs.

\section{Patients and Methods}

This was a retrospective audit of all ECVs performed at Ipswich Hospital, Ipswich during the period 2006 to Dec 2012. All ECVs were performed in birth suite. Women had cardiotocography (CTG) performed before and after the procedure. Tocolysis using $500 \mathrm{mcg}$ salbutamol by slow intravenous infusion was the routine. Ultrasound scan was used to assess fetus and to confirm presentation. Talcom powder was applied over the abdomen after which the version was by either a forward or a backward somersault. Procedure was abandoned at her request at any time during the procedure if discomfort was not tolerable. The procedure usually lasted no more than 5 minutes. Anti-D immunoglobulin was administered prophylactically to all Rhesus-negative women.

Data on demographics and on the ECV procedure and pregnancy outcome were extracted from the charts and entered into an excel spreadsheet. Data were analysed using Stata/SE 9.0 for Windows (StataCorp LP 2005). All variables were treated as categorical; continuous variables were classified into categories and treated as categorical variables. Maternal and pregnancy characteristics were compared between women with successful and unsuccessful ECV procedure using Chi squared test or Fisher's exact test, where appropriate. The association between maternal and pregnancy characteristics and successful ECV was explored using univariate logistic regression.

The study was approved by the West Moreton Hospital and Health service HREC.

\section{Results}

A total of 147 external cephalic versions (ECVs) were performed. Sixty four women (43.5\%) were in their first pregnancy. Mean (SD) BMI was 27.2 (6.4). Fifty four (36.7\%) women had a BMI of $<25 \mathrm{~kg} / \mathrm{m}^{2}, 24$ (16.3\%) were obese (BMI 30 - 34.9) and 22 (15.0\%) were morbidly obese (BMI 35 and above). Seven women (4.8\%) had had one previous caesarean section. ECV was successful in 4 of these 7 women. Eighty four (57\%) women were $<37$ weeks pregnant at the time of the ECV.

Overall ECV was successful in 79 (53.7\%) of women. Success rate was 34\% in nulliparous and $69 \%$ in multiparous women. None reverted back to breech after a successful ECV but two women with failed ECV had spontaneous version to cephalic before birth.

In Table 1 we looked at the effect of parity, BMI, placental position and gestational age on the success rate of ECV. Nulliparity was the only variable that was associated with poor success. Gestational age $<37$ weeks, anterior placenta and maternal body mass index were not associated with failure to turn the baby in our experience.

In Table 2 we looked at some of the pregnancy outcomes in the women who had an attempt at ECV. Among those with successful ECV, 32 (40.5\%) had induction of labour (IOL), in 17 (22\% of the women with successful ECV) it was for post dates. Other indications included prelabour rupture of membranes at term (5), gestational diabetes (3), pre eclampsia (2), unstable lie (1) and maternal medical concerns (4). No adverse events were recorded and no woman needed to be delivered as a result of fetal concern at the time of the ECV.

Majority of the women (83.6\%) had a vaginal birth. Intrapartum caesarean section rate in women who had had a successful ECV was $16.5 \%$. This rate was $6.5 \%$ if they went into spontaneous labour as opposed to $28 \%$ if they had IOL. Indication for caesarean section was failure to progress in two thirds of these cases. In terms of neonatal outcome there was no significant difference between the groups in terms of low Apgar score or need for admission and duration of admission into special care unit. Number of neonates with these events were however small.

\section{Discussion}

Overall success rate of $54 \%$ is similar to that reported by others in well resourced countries [4] [6]. The success 
Table 1. Univariate association between maternal and pregnancy factors and ECV outcome.

\begin{tabular}{|c|c|c|c|}
\hline Characteristics & ECV Successful $(n=79)$ & ECV unsuccessful $(n=68)$ & Odds ratio $(95 \% \mathrm{CI})$ \\
\hline \multicolumn{4}{|l|}{ Parity: } \\
\hline 0 & $22(27.9)$ & $42(61.8)$ & $\operatorname{Ref}(1.00)$ \\
\hline $1-2$ & $39(49.4)$ & $16(23.5)$ & $4.65(2.14-10.1)$ \\
\hline 3 or more & $18(22.8)$ & $10(14.7)$ & $3.44(1.36-8.70)$ \\
\hline \multicolumn{4}{|c|}{ Body Mass Index $\left(\mathrm{kg} / \mathrm{m}^{2}\right)$ : } \\
\hline$<25$ & $30(38.0)$ & $24(35.3)$ & $1.01(0.46-2.22)$ \\
\hline $25-29.9$ & 26 (32.9) & 21 (30.9) & Ref (1.00) \\
\hline $30-34.9$ & $11(13.9)$ & $13(19.1)$ & $0.68(0.25-1.83)$ \\
\hline 35 and above & $12(15.2)$ & $10(14.7)$ & $0.97(0.35-2.68)$ \\
\hline 40 and above ${ }^{*}$ & $4(5.1)$ & $4(5.9)$ & $0.85(0.21-3.55)$ \\
\hline \multicolumn{4}{|l|}{ Placental position: } \\
\hline Anterior & $30(38.0)$ & $29(44.6)$ & $0.76(0.39-1.48)$ \\
\hline Posterior/Fundal & $49(62.0)$ & $36(55.4)$ & Ref (1.00) \\
\hline \multicolumn{4}{|c|}{ Gestational age at ECV: } \\
\hline Less than 37 weeks & $43(54.4)$ & $41(60.3)$ & $0.79(0.41-1.52)$ \\
\hline 37 weeks or more & $36(45.6)$ & $27(39.7)$ & $\operatorname{Ref}(1.00)$ \\
\hline
\end{tabular}

*Referent category-less than $40 \mathrm{~kg} / \mathrm{m}^{2}$.

rate was $34 \%$ in nulliparous versus $69 \%$ in mulitiparous women. The success rate in nulliparous women was not significantly better if ECV was performed before 37 weeks as compared to 37 weeks and above, 33\% and 36\% respectively. The lower success rate in nulliparous women as been noted by others [4] [8] [11] and is thought to be due to a more tense uterus in such women [8]. It is also ones impression that nulliparous women tend not to tolerate much discomfort. Several strategies to improve success of ECV have been tested. The EECV2 Multicentre RCT [12] reported a better success rate when ECV was performed between 34 - 36 weeks compared to at 37 weeks and above but was associated with an increased risk of preterm birth and many ECVs in that study were performed nearer to 34 than to 36 weeks. We now recommend ECV at $36^{0}$ weeks in nulliparous women. Another strategy that is promising is the use of regional block. None of our cases were done with regional block. Meta analysis of several RCTs reported a better success rate (59.7\% compared with 37.6\% without analgesia; pooled relative risk 1.58; 95\% confidence interval 1.29 - 1.93) and no increase in adverse events, with the use of spinal or epidural for ECV [13].

It is of note that in women with successful ECV, vaginal birth rate was high. This supports the women's decision to undergo the ECV procedure in the first instance. Women who have spontaneous vaginal birth after successful version tend to be more satisfied with childbirth than women with planned c/section [14]. We were surprised to record a high induction of labour rate of $40.5 \%$ in the women who had had a successful ECV (our hospital overall IOL rate is around 21\%). Many of these inductions were for post dates, 53\% of women who had IOL it was for post dates, a much higher rate than that in our unit where post dates accounts for $32 \%$ of all inductions. Parity did not seem to contribute to this high IOL rate as only $22 \%$ of our nulliparous women needed IOL. This was in contrast to IOL rates of $49 \%$ in nulliparous and $30 \%$ in multiparous women reported recently in a unit in Japan [10].

There was also a higher rate of intrapartum caesarean section (16\%) compared to around 11\% for the hospital. While some [15] [16] have not seen such an increase, a meta analysis [17] of several studies has noted a two fold increase in CS rate after a successful ECV and this was independent of any increased induction rate. A recent study from Canada [18] also reported a Caesarean section rate in such women of $15 \%$ versus $6.0 \%$ in 
Table 2. Pregnancy outcome by success of external cephalic version.

\begin{tabular}{|c|c|c|c|c|}
\hline Characteristics & ECV Successful $(n=79)$ & ECV unsuccessful $(n=68)$ & Total $(n=147)$ & $p$ value \\
\hline \multicolumn{5}{|l|}{ Onset of labour } \\
\hline Spontaneous & $46(58.2)$ & $7(10.3)$ & $53(36.1)$ & $<0.000^{*}$ \\
\hline Induction of labour & $32(40.5)$ & $0(0.0)$ & $32(21.8))$ & \\
\hline No labour (Elective CS) & $0(0.0))$ & $60(88.2)$ & $60(40.8)$ & \\
\hline No labour (Emergency CS) & $1(1.3)$ & $1(1.5)$ & $2(1.4)$ & \\
\hline \multicolumn{5}{|l|}{ Mode of birth } \\
\hline Vaginal & $66(83.6)$ & $3(4.4)$ & 69 (46.9) & $<0.000^{*}$ \\
\hline Elective CS & $0(0.0)$ & $53(77.9)$ & $53(36.1)$ & \\
\hline Emergency CS & $13(16.5)$ & $12(17.7)$ & $25(17.0)$ & \\
\hline \multicolumn{5}{|l|}{ Apgar score } \\
\hline 1 min -3 or less & $4(5.1)$ & $1(1.5)$ & $5(3.4)$ & $0.374^{*}$ \\
\hline 5 min -6 or less & $2(1.4)$ & $0(0.0)$ & $2(1.4)$ & $0.499^{*}$ \\
\hline SCN admission & $11(13.9)$ & $12(17.7)$ & $23(15.7)$ & 0.536 \\
\hline \multicolumn{5}{|c|}{ Indication for SCN admission } \\
\hline Apnoea & $1(1.3)$ & $1(1.5)$ & $2(1.4)$ & $0.815^{*}$ \\
\hline Observation & $3(3.8)$ & $3(4.4)$ & $6(4.1)$ & \\
\hline Blood sugar monitoring & $2(2.5)$ & $2(2.9)$ & $4(2.7)$ & \\
\hline Congenital abnormality & $0(0.0)$ & $1(1.5)$ & $1(0.7)$ & \\
\hline Respiratory distress & $4(5.1)$ & $2(2.9)$ & $6(4.1)$ & \\
\hline Prematurity & $0(0.0)$ & $2(2.9)$ & $2(1.4)$ & \\
\hline Chorioamnionitis & $1(1.3)$ & $0(0.0)$ & $1(0.7)$ & \\
\hline Not Applicable & 68 (86.1) & 57 (83.8) & $125(85.0)$ & \\
\hline \multicolumn{5}{|l|}{ Days in SCN } \\
\hline Less than 1 day & $68(87.2)$ & $56(82.4)$ & $124(84.9)$ & $0.389^{*}$ \\
\hline 1 - 3 days & $9(11.5)$ & $8(11.8)$ & 17 (11.6) & \\
\hline More than 3 days & $1(1.3)$ & $4(5.9)$ & $5(3.4)$ & \\
\hline
\end{tabular}

*Fisher's exact; $p$ value for t-test.

women with cephalic presentation from the onset and noted that nulliparity and induction of labour were associated with caesarean section. Women who go into spontaneous labour seem to avoid the need for a caesarean birth. In our study the rate of CS was $6.5 \%$ for spontaneous labour versus $28 \%$ for labour that was induced. Similar differences in caesarean section rate was also noted previously in another study (4\% versus 34\%) [10]. The reasons for increased need for IOL and for the increase in caesarean section remain unclear [15]. Several possible reasons include inability of a breech baby to tolerate stress during labour (in which case there would be higher rate for fetal distress which we did not show (only 2 of the 13 sections were due to fetal distress), some uterine muscle abnormality that predisposed to breech presentation in the first instance and an unmolded unengaged fetal head, both of which may predispose to dystocia. This may explain our higher IOL and our higher caesarean section rate for failure to progress. Even though this increase in caesarean section rate may be small, such information is important for patient counselling and may affect a woman's preferences when choosing 
ECV.

It is also of note that we safely performed an ECV in women with one previous CS. Another study [19] also reported similar success rates in women with one previous CS compared to no previous CS. Concern about procedural success or adverse effect in women with a previous CS is unwarranted and should not deter from recommending an ECV in such women.

We provide a proactive approach to women noted to have a breech presentation during the antenatal period. ECVs were performed or supervised by a senior Obstetrician. Trainees and other colleagues could also assist or perform ECV under direct supervision and guidance. As more than $90 \%$ of ECVs were performed or supervised by a single clinician, the procedure entailed considerable homogeneity, although admittedly less generalisability.

We need to have any data regarding uptake of ECV in our unit but it has been reported to be as low as $18 \%$ [12]. We need to prospectively look at possible barriers towards ECV, including the ability or otherwise of clinicians to fully inform and consent women. Fear of pain does influence women's willingness to have ECV and whilst we promote a proactive approach when consenting we always reassure women that unbearable pain is always a reason to stop procedure. We do however emphasise that vast majority of women report that the experienced pain is bearable. Use of videos as part of the information on ECV is another area that needs further research. If women with breech presentation have a primary caesarean section in the first pregnancy a large number will have an elective repeat procedure in their second pregnancy. Data [20] regarding increased risk of placenta accrete after CS are concerning.

\section{Conclusion}

We have confirmed that ECV is a safe procedure with a success rate that is comparable to many other studies but suggested more research into other novel methods to increase uptake further and to improve success rates especially in nulliparous women. Mothers with successful ECV do have a higher IOL and a higher CS rate especially in women whose labour is induced.

\section{Acknowledgements}

We would like to acknowledge the help of the medical records Unit at Ipswich hospital for identification and retrieval of charts and to Donna Mason to assist with data cleansing.

\section{References}

[1] Hickok, D., Gordon, D., Milberg, J., Williams, M.A., et al. (1992) The Frequency of Breech Presentation by Gestational Age at Birth: A Large Population-Based Study. American Journal of Obstetrics \& Gynecology, 166, 851-852. http://dx.doi.org/10.1016/0002-9378(92)91347-D

[2] Hannah, M.E., Hannah, W.J., Hewson, S.A., Hodnett, E.D., et al. (2000) Planned Caesarean Section versus Planned Vaginal Birth for Breech Presentation at Term: A Randomised Multicentre Trial. Term Breech Trial Collaborative Group. Lancet, 356, 1375-1383. http://dx.doi.org/10.1016/S0140-6736(00)02840-3

[3] Hutton, E.K., Hannah, M.E. and Barrett, J. (2002) Use of External Cephalic Version for Breech Pregnancy and Mode of Delivery for Breech and Twin Pregnancy: A Survey of Canadian Practitioners. Journal of Obstetrics and Gynaecology Canada, 24, 804-810.

[4] Hofmeyr, G.J. and Kulier, R. (2005) External Cephalic Version for Breech Presentation at Term. Cochrane Database of Systematic Reviews, 1, CD000083. http://dx.doi.org/10.1002/14651858.CD000083

[5] Royal College of Obstetricians and Gynaecologists (2006) The Management of Breech Presentation. Guideline No. 20b. Royal College of Obstetricians and Gynaecologists Clinical Green Top Guidelines.

[6] External Cephalic Version and Reducing the Incidence of Breech Presentation (2006) Green Top Guidelines No.20a. RCOG Press, London.

[7] American College of Obstetrics and Gynecology (ACOG) (2000) Clinical Management Guidelines for ObstetricianGynecologists: External Cephalic Version. ACOG Practice Bull, 13, 380-385.

[8] Kok, M., Cnossen, J., Gravendeel, L., Van Der Post, J.A., et al. (2008) Clinical Factors to Predict the Outcome of External Cephalic Version: A Metaanalysis. American Journal of Obstetrics \& Gynecology, 199, 630.e1-630.e7. http://dx.doi.org/10.1016/j.ajog.2008.03.008

[9] Benmeir, A., Erez, Y., Sela, H.Y., Shveiky, D., et al. (2008) Prognostic Parameters for Successful External Cephalic Version. Journal of Maternal-Fetal and Neonatal Medicine, 21, 660-662. 
http://dx.doi.org/10.1080/14767050802244938

[10] Cho, L.Y., Lau, W.L., Lo, T.K., Tang, H.H., et al. (2012) Predictors of Successful Outcomes after External Cephalic Version in Singleton Term Breech Pregnancies: A Nine Year Historical Cohort Study. Hong Kong Medical Journal, 18, 11-19.

[11] Obeidat, N., Lataifeh, I., Al-Khateeb, M., Zeyed, F., et al. (2011) Factors Associated with the Success of External Cephalic Version of Breech Presentation at Term. Clinical and Experimental Obstetrics and Gynecology, 38, $386-389$.

[12] Hutton, E.K., Hannah, M.E., Ross, S.J., Delisle, M.F., et al. (2011) Early External Cephalic Version (ECV) 2 Trial: An International Multicentre Randomised Controlled Trial of Timing of ECV for Breech Pregnancies. British Journal of Obstetrics and Gynaecology, 118, 564-577. http://dx.doi.org/10.1111/j.1471-0528.2010.02837.x

[13] Goetzinger, K.R., Harper, L.M., Tuuli, M.G., Macones, G.A., et al. (2011) Effect of Regional Anesthesia on the Success Rate of External Cephalic Version: A Systematic Review and Meta-Analysis. Obstetrics \& Gynecology, 118, 1137-1144. http://dx.doi.org/10.1097/AOG.0b013e3182324583

[14] Bogner, G., Hammer, B.E., Schausberger, C., Fischer, T., et al. (2013) Patient Satisfaction with Childbirth and External Cephalic Version. Archives of Gynecology and Obstetrics, 289, 523-531.

[15] Kabiri, D., Elram, T., Aboo-dia, M., Elami-Suzin, M. et al. (2011) Timing of Delivery after External Cephalic Version and the Risk for Cesarean Delivery. Obstetrics \& Gynecology, 118, 209-213. http://dx.doi.org/10.1097/AOG.0b013e31822545a9

[16] Clock, C., Kurtzman, J., White, J. and Chung, H. (2009) Cesarean Risk after Successful External Cephalic Version: A Matched, Retrospective Analysis. Journal of Perinatology, 29, 96-100. http://dx.doi.org/10.1038/jp.2008.227

[17] Chan, L., Tang, J., Tsoi, K., Fok, W.Y., et al. (2004) Intrapartum Cesarean Delivery after Successful External Cephalic Version: A Meta-Analysis. Obstetrics \& Gynecology, 104, 155-160. http://dx.doi.org/10.1097/01.AOG.0000129240.54949.d2

[18] Kuppens, S.M.I., Hutton, E.K., Hasaart, T.H.M., et al. (2012) Mode of Delivery Following Successful External Cephalic Version: Comparison with Spontaneous Cephalic Presentations at Delivery. Journal of Obstetrics and Gynaecology Canada, 35, 883-888.

[19] Abenhaim, H.A., Varin, J. and Boucher, M. (2009) External Cephalic Version among Women with a Previous Cesarean Delivery: Report on 36 Cases and Review of the Literature. Journal of Perinatal Medicine, 37, 156-160. http://dx.doi.org/10.1515/JPM.2009.006

[20] Higgins, M.F., Monteith, C., Foley, M. and O’Herlihy, C. (2013) Real Increasing Incidence of Hysterectomy for Placenta Accreta Following Previous Caesarean Section. The European Journal of Obstetrics \& Gynecology and Reproductive Biology, 19, S0301-S2115. 